\title{
ATIVIDADE ANTICOLINESTERÁSICA E PERFIL QUÍMICO DE UMA FRAÇÃo CROMATOGRÁFICA ATIVA DO EXTRATO ETANÓLICO DAS FLORES Bellis perennis L. (Asteraceae)
}

\author{
Thiago Henrique Costa Marques, Pauline Sousa dos Santos e Rivelilson Mendes de Freitas* \\ Departamento de Bioquímica e Farmacologia, Centro de Ciências da Saúde, Universidade Federal do Piauí, 64049-550 Teresina \\ - PI, Brasil \\ Rusbene Bruno Fonseca de Carvalho e Cassio Herbert Santos de Melo \\ Departamento de Química, Centro de Ciências da Natureza, Universidade Federal do Piauí, 64049-550 Teresina - PI, Brasil \\ Juceni Pereira David \\ Faculdade de Farmácia, Universidade Federal da Bahia, 40170-115 Salvador - BA, Brasil \\ Jorge Mauricio David e Luciano Silva Lima \\ Instituto de Química, Universidade Federal da Bahia, 40170-115 Salvador - BA, Brasil
}

Recebido em 2/7/12; aceito em 14/11/12; publicado na web em 21/2/13

\begin{abstract}
ANTICHOLINESTERASE ACTIVITY AND CHEMICAL PROFILE OF AN ACTIVE CHROMATOGRAPHIC FRACTION OF ETHANOLIC EXTRACT FROM Bellis perennis L. (Asteraceae) FLOWERS. This work describes the isolation of an active flavonoid fraction and identification of isorhamnetin 3-O-B-D-(6"-acetyl)-galactopyranoside from flowers of $B$. perennis, and also the evaluation of anticholinesterase (AChE) activity of ethanolic extract from flowers (EEF) and the active fraction. The chemical structure of the flavonoid was defined on the basis of spectroscopic ${ }^{1} \mathrm{H}$ NMR, IR and UV data. EEF or flavonoid reduces AChE activity in vivo, while flavonoid also reduces AChE activity in vitro, showing a value of $1.49 \mu \mathrm{M}$ for $50 \%$ inhibitory concentration $\left(\mathrm{IC}_{50}\right)$, suggesting potential use as an insecticide or in the treatment of neurodegenerative diseases such as Alzheimer's disease.
\end{abstract}

Keywords: anticholinesterase activity; B. perennis; isorhamnetin 3-O- $\beta$-D-(6"-acetyl)-galactopyranoside.

\section{INTRODUÇÃO}

Desde o inicio da civilização humana, as plantas têm sido utilizadas com fins medicinais. ${ }^{1}$ Dentre as várias famílias de plantas medicinais, podem ser destacadas plantas com potencial biológico, como a família Asteraceae, que compreende, aproximadamente, 1500 gêneros e 2300 espécies incluindo o gênero Bellis e a espécie Bellis perennis L., conhecida como margarida comum ou margarida inglesa. ${ }^{2} \mathrm{O}$ estudo fitoquímico das raízes e flores de $B$. perennis demonstrou a presença de várias moléculas bioativas, que explica a sua ampla utilização farmacológica. Dentre estas biomoléculas, pode ser verificada a presença de várias saponinas e flavonoides. ${ }^{2}$ Avato e Tava $^{3}$ descreveram a presença de compostos voláteis na composição química dos óleos essenciais das folhas e flores desta espécie, no entanto estes compostos têm sido pouco investigados.

Trabalhos anteriores utilizando $B$. perennis relatam propriedades expectorante, cicatrizante, anti-inflamatória, anti-hemorrágica, anestésica, antiparasitária, antifúngica, antimicrobiana, anticonvulsivante e antioxidante. ${ }^{3,4}$ Dentre as atividades relevantes ao tratamento de doenças neurodegenerativas, é crescente a busca de novos inibidores da acetilcolinesterase (AChE) em extratos de plantas, envolvendo principalmente plantas medicinais já utilizadas na medicina tradicional no tratamento de várias patologias. ${ }^{5}$ Faz-se necessária a investigação dos efeitos de compostos ativos extraídos de plantas, uma vez que estes poderão fornecer novos subsídios para o tratamento clínico das doenças neurodegenerativas, como a doença de Alzheimer.

O Mal de Alzheimer é uma doença cerebral degenerativa que compromete inicialmente a memória e, posteriormente, a capacidade de raciocínio e a comunicação. ${ }^{6,7} \mathrm{O}$ tratamento consiste na tentativa de restauração da função colinérgica. ${ }^{8}$ Inibidores de AChE são amplamente utilizados no tratamento dessa patologia

*e-mail: rivelilson@pq.cnpq.br por meio da modulação da atividade das enzimas que degradam a acetilcolina. ${ }^{6,9}$ As áreas cerebrais patologicamente mais afetadas no Mal de Alzheimer são o hipocampo e o neocórtex. Estas áreas estão associadas às funções do sistema nervoso central de forma mais predominante. ${ }^{10}$

Os mecanismos antioxidantes hipocampais são importantes no processo de neuroproteção contra várias desordens neurológicas. ${ }^{11} \mathrm{O}$ efeito amnésico causado por lesões hipocampais é bem documentado, levando a numerosas teorias de função hipocampal sobre memória. Um número crescente de estudos sugere que efeitos comportamentais diversificados devem ser associados a diferentes sub-regiões hipocampais. Por exemplo, o hipocampo dorsal tem papel preferencial em certas formas de aprendizagem e memória, que são notadamente afetadas na Doença de Alzheimer. ${ }^{12}$

Descreveu-se no presente trabalho o isolamento e a identificação de um flavonoide, isorhamnetina 3-O- $\beta$-D-(6" -acetil)galactopiranosideo, das flores de $B$. perennis e, ainda, a avaliação de atividade anticolinesterásica do extrato etanólico das flores (EEF) e da fração enriquecida de flavonoides isolada. A estrutura química da substância isolada foi definida com base na análise de dados espectroscópicos de $\mathrm{RMN}{ }^{1} \mathrm{H}$ e ultravioleta e a comparação dos dados obtidos com os registrados na literatura.

\section{PARTE EXPERIMENTAL}

\section{Material vegetal}

As flores da planta medicinal B. perennis L. para o referido estudo foram coletadas em outubro de 2010 pela manhã (07:00) na Fazenda Sítio São José localizada no município de Pacoti, na região do Maciço de Baturité, Ceará. A exsicata da espécie (Número 27.276) foi depositada no Herbário Graziella Barroso, da Universidade Federal do Piauí, e identificada pela Profa. Dra. R. F. M. de Barros. A coleta das 
flores foi feita manualmente, sendo, então, lavadas em água corrente, seguida de água destilada.

\section{Procedimento para obtenção dos extratos e isolamento do constituinte químico}

O material vegetal (310 g) foi seco à temperatura ambiente, macerado estaticamente e extraído com etanol. O extrato etanólico obtido permaneceu 7 dias em repouso, agitando-se duas vezes ao dia, sendo em seguida concentrado por evaporador rotatório sob pressão reduzida para obtenção do extrato bruto. Este foi submetido à extração a frio com solventes de polaridade crescente, obtendo-se as seguintes frações e seus respectivos rendimentos: hexano (57\%), clorofórmio (19\%), acetato de etila $(0,3 \%)$ e água $(10,85 \%)$. Estas frações foram evaporadas sob pressão reduzida e cromatografadas separadamente em coluna de sílica gel, monitoradas por cromatografia em camada delgada. A fração hexânica $(19,95 \mathrm{~g})$ foi adsorvida em gel de sílica e submetida à coluna cromatográfica, utilizando-se os eluentes metanol/água (F-11/12) nas proporções de 95:5. Depois de liofilização, obtiveram-se $55 \mathrm{mg}$ de um líquido marrom viscoso com característica de substância pura em CCD que se constatou, por RMN ${ }^{1} \mathrm{H}$ e IV, tratar-se de uma substância identificada como isorhamnetina 3-O-B-D-(6"-acetil)-galactopiranosídeo (Figura 1).

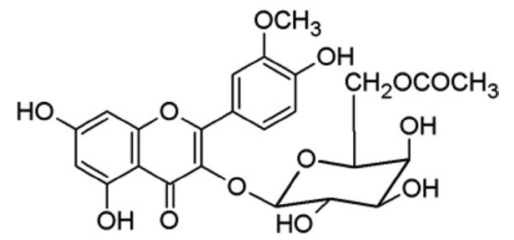

Figura 1. Estrutura química da isorhamnetina 3-O- $\beta-D-(6$ "-acetil)-galactopiranosídeo detectada em EEF

\section{Análise da fração isolada do EEF de B. perennis}

\section{Equipamentos utilizados}

Nas análises cromatográficas, utilizou-se um sistema de cromatografia líquida de alta eficiência (CLAE) composto de injetor automático, bloco controlador da temperatura da coluna e detector de arranjo de diodos (DAD) responsável pelo registro dos espectros no UV da fração de flavonoides, modelo Ultimate 3000 da Dionex. Os espectros de $\mathrm{RMN}$ de ${ }^{1} \mathrm{H}$ foram registrados em equipamento marca Varian mod. Gemini 2000 operando a $300 \mathrm{MHz}$, utilizando o resíduo do solvente não deuterado como padrão interno em referência ao TMS. O espectro no IV foi registrado em equipamento da Shimadzu IRAffinity-1 utilizando-se pastilhas de $\mathrm{KBr}$.

\section{Condições cromatográficas}

A amostra foi filtrada em unidade filtrante $0,45 \mu \mathrm{m}$ (Millipore, EUA) e, em seguida, $10 \mu \mathrm{L}$ da solução da amostra foram aplicados no sistema com auxílio de injetor automático. Nas análises cromatográficas, empregou-se um sistema cromatográfico CLAE-DAD, sob as seguintes condições: coluna fase reversa C18 (3,5 $\mu \mathrm{m}, 2,1 \mathrm{x}$ $75 \mathrm{~mm}$ ) Elipse, volume de injeção $10 \mu \mathrm{L}$; como fase móvel MeOH/ água acidificada com ácido acético $(0,01 \%)$ na proporção de $2: 3$, eluição isocrática, fluxo $0,5 \mathrm{~mL} / \mathrm{min}, 27^{\circ} \mathrm{C}$ e comprimento de onda do detector em $\lambda=330 \mathrm{~nm}$.

\section{Determinação in vitro da atividade da enzima acetilcolinesterase}

$\mathrm{O}$ efeito inibitório da substância isolada sobre a atividade da enzima acetilcolinesterase in vitro foi avaliada como descrito por Ellman e colaboradores ${ }^{13}$ com algumas modificações, conforme detalhado por Moyo e colaboradores. ${ }^{14}$
Para a atividade de inibição quantitativa, um espectrômetro Biospectro SP 220 foi utilizado. Inicialmente, $100 \mu \mathrm{L}$ da amostra em triplicata nas concentrações de 0,0125; 0,00652; 0,003125 e $0,001563 \%$ em solução tampão $50 \mathrm{mM}$ Tris- $\mathrm{HCl}, \mathrm{pH}$ 8, e metanol $10 \%$ foram misturados com $100 \mu \mathrm{L}$ de AChE $(0,22 \mathrm{U} / \mathrm{mL}$ em $50 \mathrm{mmol} / \mathrm{L}$ Tris-HCl, tampão pH $8,0,1 \%$ BSA) e $200 \mu \mathrm{L}$ de tampão (50 mmol/L Tris-HCl, pH 8, 0,1\% BSA). A mistura foi incubada por $5 \mathrm{~min}$ a $30{ }^{\circ} \mathrm{C}$. Em seguida, $500 \mu \mathrm{L}$ de ácido ditiobisnitrobenzoico (DTNB) (3 mmol/L em Tris- $\mathrm{HCl}, \mathrm{pH} 8,0,1 \mathrm{~mol} / \mathrm{L}$ de $\mathrm{NaCl}$, $0,02 \mathrm{~mol} / \mathrm{L} \mathrm{MgCl}_{2}$ ) e $100 \mu \mathrm{L}$ de iodeto de acetiotiocolina (ATCI) (4 mmol/L em água) foram adicionados.

O teste branco foi realizado também em triplicata pela substituição da enzima AChE por $100 \mu \mathrm{L}$ de tampão $(50 \mathrm{mmol} / \mathrm{L}$ Tris- $\mathrm{HCl}, \mathrm{pH} 8$, $0,1 \%$ BSA). A reação foi monitorada por $5 \mathrm{~min}$ em $412 \mathrm{~nm}$. O ensaio é baseado na medição espectrofotométrica do aumento da cor amarela produzida a partir de tiocolina quando esta reage com o íon ditiobisnitrobenzoato. O fármaco rivastigmina $\left(\right.$ Exelon $\left.^{\circledR}\right)$ foi usado como droga padrão (controle positivo) e como controle negativo foi utilizado o Tampão (0,1\% de metanol em 50 mmol/L Tris-HCl, pH 8, 10\%).

As porcentagens de inibição da substância isolada e da rivastigmina foram calculadas de acordo com a seguinte equação: I $(\%)=$ 1 - (taxa de reação da amostra/taxa de reação do branco)*100. Os valores da concentração inibitória média $\left(\mathrm{CI}_{50}\right)$ foram obtidos por intermédio de plotagem Log-Probit.

\section{Animais}

Foram utilizados camundongos machos albinos (Mus musculus), variedade Swiss, adultos, com 2 meses de idade e peso variando de 25 a 30 g, provenientes do Biotério Central do Centro de Ciências Agrárias da Universidade Federal do Piauí .

Durante todos os experimentos, os animais foram aclimatados a $25 \pm 2{ }^{\circ} \mathrm{C}$ e mantidos em gaiolas de acrílico apropriadas, com no máximo 6 animais. Os animais foram observados durante $24 \mathrm{~h}$ em condições ambientais semelhantes, com ciclo claro/escuro alternado de $12 \mathrm{~h}$, recebendo ração padrão tipo Purina e água ad libitum. Os experimentos foram realizados de acordo com o guia de cuidados e usos de animais de laboratório do Departamento de Saúde e Serviços Humanos dos Estados Unidos da América.

Todos os experimentos desenvolvidos neste estudo foram aprovados pelo Comitê de Ética em Experimentação com Animais da UFPI (CEEA/UFPI n077/10). Os procedimentos referentes à eutanásia dos animais estavam em conformidade com o Parágrafo Único do Artigo $2^{\circ}$ da Resolução N ${ }^{\circ} 714$, de 20 de Junho de 2002, do Conselho Federal de Medicina Veterinária - CFMV.

\section{Tratamento dos animais}

Os animais foram divididos em 6 grupos de 7 animais. O primeiro grupo foi tratado com Tween 80 0,05\% dissolvido em solução salina $0,9 \%$ (i.p.; $\mathrm{n}=7$; controle negativo). $\mathrm{O}$ grupo controle positivo foi tratado com rivastigmina (400 mg/kg, i.p.; $\mathrm{n}=7$ ). Três outros grupos foram tratados com EEF nas doses de 50,100 e $150 \mathrm{mg} / \mathrm{kg}$ (i.p.; $\mathrm{n}=7$ por grupo) respectivamente. Por sua vez, o último grupo foi tratado com a fração enriquecida de flavonoides dissolvida em Tween 80 0,05\% dissolvido em solução salina $0,9 \%$ na dose de 10 $\mathrm{mg} / \mathrm{kg}$ (i.p.; $\mathrm{n}=7$ ).

Após 24 h de observação, todos os animais que sobreviveram a esse período de observação foram anestesiados pela administração de pentobarbital sódico (10-15 mg/100 g de peso, i.p.). Os animais foram eutanasiados por decapitação, os encéfalos rapidamente retirados e o hipocampo dissecado para preparação do homogenato a $10 \% \mathrm{em}$ tampão fosfato $(\mathrm{pH} 7,4)$. 


\section{Determinação in vivo da atividade da enzima acetilcolinesterase}

A atividade acetilcolinesterásica foi determinada segundo o método descrito por Ellman e colaboradores,${ }^{13}$ tendo como princípio básico a medida da velocidade de produção de tiocolina à proporção que a acetiltiocolina (ATC), utilizada como substrato, foi hidrolisada.

Isto foi acompanhado pela reação contínua do tiol com o íon 5:5-ditiobis-2-nitrobenzoato (I) para produzir o ânion amarelo do ácido 5-tio-2-nitro-benzoico (II), cuja absorbância foi medida em 412 nm, em um espectrofotômetro Biospectro SP 220, o que permitiu leituras automáticas em sistema digital e forneceu maior sensibilidade.

A atividade enzimática foi medida pela leitura da variação da absorbância por minuto, durante $3 \mathrm{~min}$, sendo a reação linear durante pelo menos $10 \mathrm{~min}$. A atividade específica foi expressa em nanomoles de ATC hidrolisados por miligrama de proteína por minuto (nmol/mg proteína/min).

Os tecidos hipocampais $(n=7)$ de todos os grupos foram homogeneizados em tampão fosfato ( $\mathrm{pH} \mathrm{8,0;0,1} \mathrm{M)} 10 \%$ e o homogenato $(10 \mu \mathrm{L})$ foi adicionado a uma cubeta contendo $500 \mu \mathrm{L}$ do tampão fosfato (pH 7,4), $890 \mu \mathrm{L}$ de água destilada e $50 \mu \mathrm{L}$ de ácido ditiobisnitrobenzoico (DTNB) 0,01 M e a absorbância zerada. Após a absorbância ser deixada em zero, a cubeta foi retirada e a ela foi acrescentado iodeto de acetiotiocolina 0,075 M. A absorbância foi registrada por $3 \mathrm{~min}$ em $412 \mathrm{nM}$. A atividade da enzima foi calculada como modificações na absorbância do min 3 para o min 0 , relativo ao conteúdo de proteína contido no homogenato. ${ }^{15} \mathrm{O}$ procedimento completo foi feito usando-se um espectrofotômetro Biospectro SP 220 ajustado para um comprimento de onda de $412 \mathrm{~nm}$.

\section{Análise estatística}

Os resultados obtidos foram expressos como média \pm erro padrão da média (E.P.M). Para avaliar a significância das diferenças entre as médias, utilizou-se Análise de Variância (ANOVA One-way) seguido do $t$-Student-Neuman-Keuls como post hoc teste. As diferenças entre as médias foram consideradas significativas quando o valor obtido para " $p$ " foi menor que $0,05(p<0,05)$. A análise estatística foi realizada utilizando o programa estatístico Graph-PadPrism ${ }^{\circledR} 5.01$ (GraphPadSoftware, San Diego, CA, E.U.A.).

\section{RESULTADOS E DISCUSSÃO}

\section{Análise da composição flavanoídica da fração obtida de EEF}

A análise dos espectros de RMN de ${ }^{1} \mathrm{H}$ e do espectro no IV permitiu concluir que a fração é formada por uma mistura de substâncias fenólicas e glicosiladeos. No espectro IV só podem ser observados sinais de estiramento $\mathrm{O}-\mathrm{H}$, e C-O de alcoóis e fenóis além de estiramento $\mathrm{C}=\mathrm{O}$ de fenil cetonas e éster. Já o espectro de $\mathrm{RMN}$ de ${ }^{1} \mathrm{H}$ (Figura 2) foi mais informativo. As ressonâncias registradas entre d 6,2 a 8,0 ppm são características de hidrogênios ligados a anel aromático. Os dubletos observados em d 6,2 e 6,5 $(J=2,1 \mathrm{~Hz})$ são indicativos de hidrogênios do anel A de flavonoides 6,7-dissubstituídos e o singleto em d 8,5 foi identificado como $\mathrm{OH}$ quelado de flavonóis. Pode-se verificar também em d 6,9 e 7,5 a presença de sinais de sistemas AMX indicando a presença também de anel B 1,3,4-trissubstituídos e dubletos $(J=8,7 \mathrm{~Hz})$ característicos de flavonoides com anel B 1,4-dissubstituído. Na região de d 3,0-4,2 ppm pode-se verificar a presença de vários multipletos, indicando a presença de hidrogênios oximetínicos e oximetilênicos de unidades glicosídicas. Pode-se concluir que os açúcares estão ligados aos flavonoides devido ao sinal do carbono anomérico em d 5,2 $(J=8,2 \mathrm{~Hz})$.

A isorhamnetina 3-O- $\beta$-D-(6"-acetil)-galactopiranosídeo (Figura 1) foi identificada na fração enriquecida isolada de EEF, e, pelo espectro no UV, foi eluída no Tr correspondente a 7,2 min no cromatograma

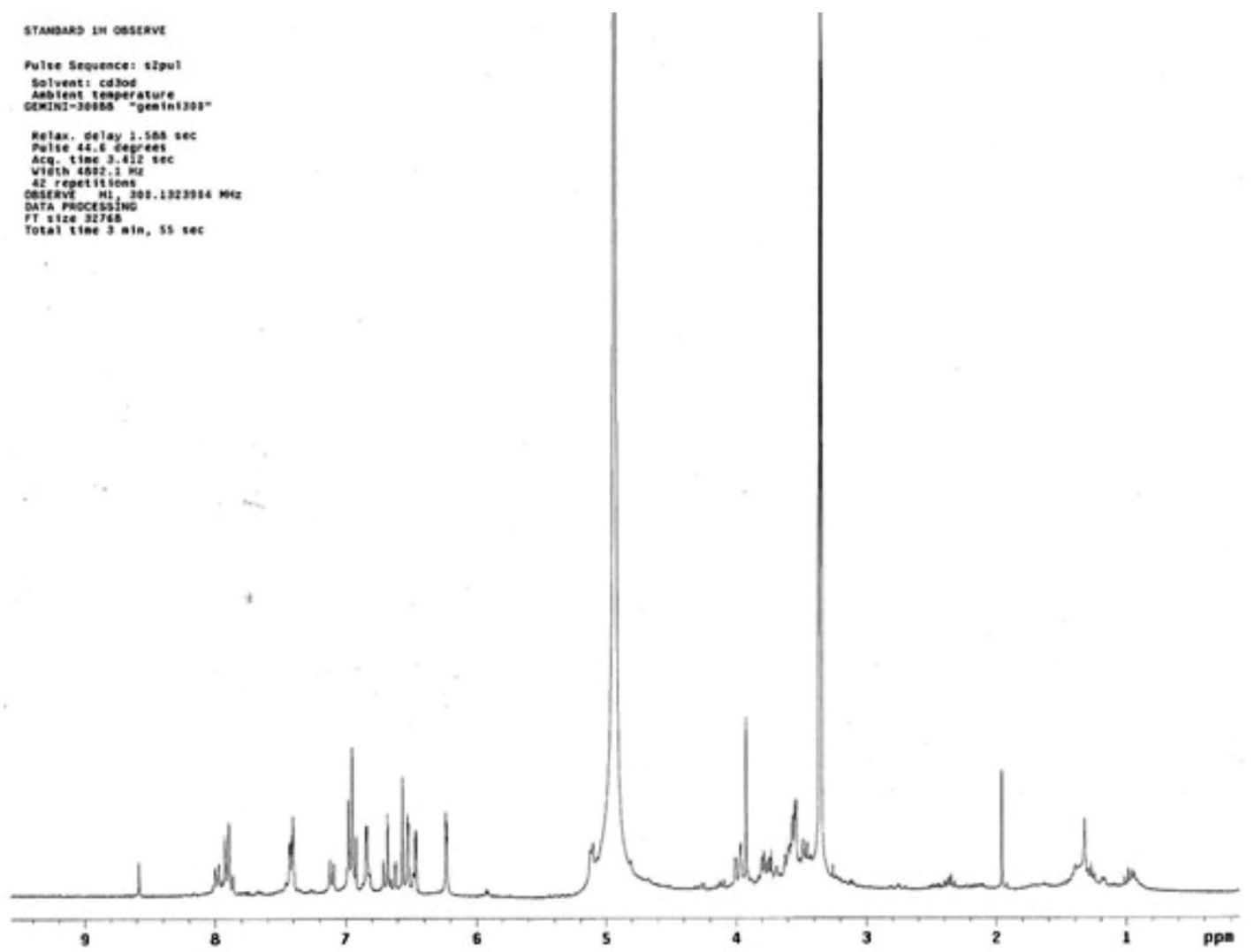

Figura 2. Espectro de $\mathrm{RMN} \mathrm{de}{ }^{l} \mathrm{H}\left[300 \mathrm{MHz}, C D_{6} \mathrm{OD}, d\right.$ (ppm)] 
registrado (Figura 3). Em estudos anteriores com B. perennis, foram isolados vários flavonoides glicosilados tais como rutina, apigenina 7-O-glicopiranosídeo, isoramentina 3-O-glicopiranosídeo, ${ }^{16}$ bem como a isorhamnetina 3-O-B-D-(6"-acetil)-galactopiranosídeo e caempferol 3-O- $\beta$-D-glicopiranosídeo, ${ }^{17}$ entre outros. A isorhamnetina pode ser identificada na fração ativa pelos sinais característicos observados no espectro de RMN de ${ }^{1} \mathrm{H}$. Pode-se verificar a presença de um grupo metoxilíco $(\mathrm{d} 3,92)$ e um grupo acila em d 2,0, ambos registrados como singletos, além dos sinais de dois hidrogênios oximetilênicos que se encontram deslocados entre d 4,2-4,4 indicando a localização onde o grupo acetil estava esterificado. Estes dados, juntamente com os sinais anteriormente descritos, sugerem a presença de isorhamnetina 3-O- $\beta$-D-(6"-acetil)-galactopiranosídeo como um dos componentes da fração e derivados de caempferol. No espectro de RMN não foram registrados sinais de $\mathrm{H}-3$ de flavonas, sugerindo assim que na fração não existem derivados de apigenina.

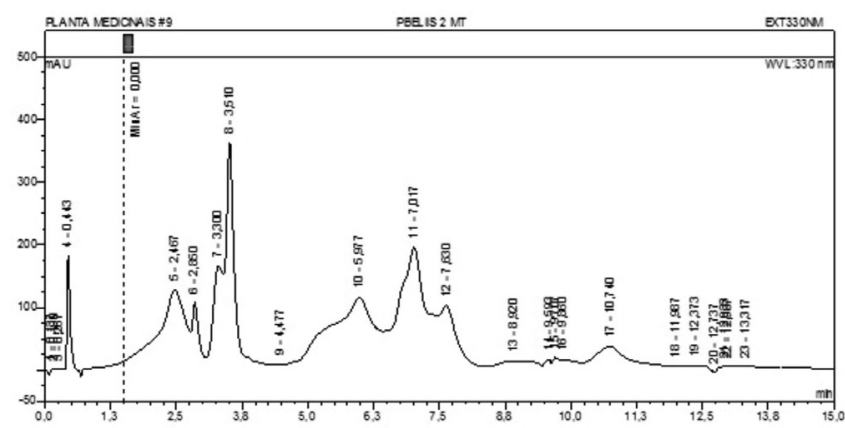

Figura 3. Cromatograma da fase reversa $C-18$ da fração isolada de EEF a $27^{\circ} \mathrm{C}$ e detector a $330 \mathrm{~nm}$

A análise no CLAE da fração enriquecida isolada do EEF (Figura 3) corroborou as conclusões dos espectros de RMN e no IV uma vez que, pelos espectros no UV registrados na eluição dos componentes da mistura, se pode também concluir que esta fração é composta de uma mistura de vários compostos fenólicos com tempos de retenção muito próximos. As análises dos espectros de UV gerados pelo DAD permitem concluir que todos os principais componentes da mistura são flavonoides, uma vez que apresentam bandas características nas faixas de 25-260 e 330-350 nm (Figura 1S, material suplementar), características de flavonoides. O perfil do espectro no UV da substância eluída em 7,2 min é próximo ao de isorhamnetina 3-O-ß-D-(6" -acetil)-galactopiranosídeo, corroborando, assim, os sinais registrados nos espectros de RMN.

\section{Determinação in vitro da atividade anticolinesterásica da fração enriquecida de flavonoides isolada do EEF de $B$. perennis}

Nos estudos in vitro, verificou-se uma inibição da atividade da $\mathrm{AChE}$ de 83,85 e 17,22 quando se usou a rivastigmina (Exelon ${ }^{\circledR}$ ) como controle positivo nas concentrações de 0,2 e $0,0125 \%$, respectivamente. A fração enriquecida de flavonoides isolada do EEF, nas concentrações de 0,$0125 ; 0,00652 ; 0,003125$ e $0,001563 \%$, produziu uma inibição de 64,30; 55,83; 43,87 e 38,13\% na atividade da AChE, respectivamente. Com base nesses resultados, também foi determinada a concentração inibitória $50 \%\left(\mathrm{CI}_{50}\right)$, que correspondeu a 3,1 $\mu \mathrm{M}$, variando de 1,7 a $6,1 \mu \mathrm{M}$ com intervalo de confiança de $95 \%$.

Um estudo realizado anteriormente com flavonoides demonstrou atividade anticolinesterásica in vitro para esses compostos. Entre esses flavonoides, a quercetina e a macluraxantona apresentaram um valor de $\mathrm{CI}_{50}$ para a atividade de AChE correspondente a 353,86 e 8,47 $\mu \mathrm{M}$, respectivamente. No entanto, já os flavonoides rutina e caempferol

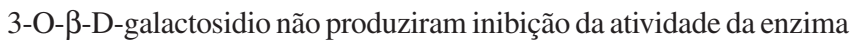
acetilcolinesterase. ${ }^{18}$ Nessa perspectiva, a fração enriquecida com o flavonoide isorhamnetina 3-O- $\beta$-D-(6"-acetil)-galactopiranosídeo isolada do EEF apresentou $\mathrm{CI}_{50}$ menor que os outros flavonoides já descritos na literatura para atividade da AChE.

A enzima inibida pelos referidos compostos e/ou extratos pode ser instável e a sua regeneração é relativamente rápida quando comparada com outros compostos. Assim, os praguicidas podem ser menos perigosos com relação à exposição humana, já que se avalia a toxicidade aguda em camundongos por meio de estudos pré-clínicos e se detecta um perfil de segurança, podendo ser utilizado em ambientes domésticos e/ou hospitalares sem prejudicar a saúde humana.

Sugere-se, portanto, que o composto pode aumentar de forma mais eficaz a estimulação colinérgica e produzir um efeito inseticida mais potente do que outros inseticidas, como carbamatos e organofosforados, para aplicação na agropecuária ou em ambientes domésticos.

\section{Determinação in vivo da atividade anticolinesterásica do EEF e da fração enriquecida de flavonoides (composto)}

Complementando a análise dos efeitos inibitórios sobre a atividade da AChE, nesse estudo foi verificada uma diminuição de 81 , 83 e $82 \%$ após o tratamento com as doses de $50 \mathrm{mg} / \mathrm{kg}(1,91 \pm 0,25$; $\mathrm{p}<0,05), 100 \mathrm{mg} / \mathrm{kg}(1,66 \pm 0,21 ; \mathrm{p}<0,05)$ e $150 \mathrm{mg} / \mathrm{kg}(1,79 \pm 0,13$; $\mathrm{p}<0,05)$ do EEF em relação ao controle negativo $(10,03 \pm 0,16$; $\mathrm{p}<0,05)$, respectivamente. Da mesma forma, em comparação ao grupo controle positivo tratado com rivastigmina $(5,69 \pm 1,20)$ verificou-se uma redução de 67, 71 e 69\% na atividade da AChE entre os camundongos tratados com as doses de $50 \mathrm{mg} / \mathrm{kg}(1,91 \pm 0,25$; $\mathrm{p}<0,05), 100 \mathrm{mg} / \mathrm{kg}(1,66 \pm 0,21 ; \mathrm{p}<0,05)$ e $150 \mathrm{mg} / \mathrm{kg}(1,79 \pm$ $0,13 ; \mathrm{p}<0,05)$ do EEF, respectivamente.

Além disso, foi verificada uma diminuição de $91 \%$ na atividade da $\mathrm{AChE}$ entre os camundongos tratados com a dose de $10 \mathrm{mg} / \mathrm{kg}$ $(0,89 \pm 0,21)$ da fração enriquecida de flavonoides isolada do EEF de B. perennis em relação ao controle negativo $(10,03 \pm 0,16 ; \mathrm{p}<0,05)$. Em comparação ao grupo controle positivo tratado com rivastigmina $(5,69 \pm 1,20)$ verificou-se uma redução de $84,4 \%$ na atividade da AChE entre os camundongos tratados com a dose de $10 \mathrm{mg} / \mathrm{kg}$ $(0,89 \pm 0,21 ; p<0,05)$ do composto isolado. A fração enriquecida de flavonoides isolada do EEF também produziu uma redução de 53,4; 46,4 e 50,3\% na atividade da AChE entre os camundongos tratados com a dose de $10 \mathrm{mg} / \mathrm{kg}(0,89 \pm 0,21)$ em relação aos grupos tratados com as doses de $50 \mathrm{mg} / \mathrm{kg}(1,91 \pm 0,25 ; \mathrm{p}<0,05), 100 \mathrm{mg} / \mathrm{kg}$ $(1,66 \pm 0,21 ; \mathrm{p}<0,05)$ e $150 \mathrm{mg} / \mathrm{kg}(1,79 \pm 0,13 ; \mathrm{p}<0,05)$ do EEF de $B$. perennis, respectivamente (Figura 4).

A farmacodinâmica de uma determinada substância pode ser quantificada pela relação entre a dose do composto e a resposta observada após a sua administração. Dessa forma, intuitivamente, o esperado é que a relação dose-resposta esteja estreitamente relacionada com a ligação entre o composto e o receptor, e pode ser verificado que isso pode realmente ocorrer por meio de muitas combinações entre esses. ${ }^{19}$ Por conseguinte, foi verificado que a inibição da atividade da $\mathrm{AChE}$ produzida pelo EEF não é proporcional à concentração de enzimas que estão ligadas pelo extrato, uma vez que não foi possível verificar um efeito dose-dependente entre as diferentes doses do extrato avaliado in vivo. De acordo com a pressuposição anterior, pode-se suger que o EEF apresentou eficácia na inibição da atividade da AChE, uma vez que pode ser considerado, a partir dos resultados, que a inibição da atividade da AChE foi significativa. No entanto, um aumento das doses do EEF não produziu uma resposta inibitória adicional significativa. Esse estado pode ter sido habitualmente alcançado devido à ocupação de todos os sítios alvos da enzima responsável pelo efeito do extrato. 


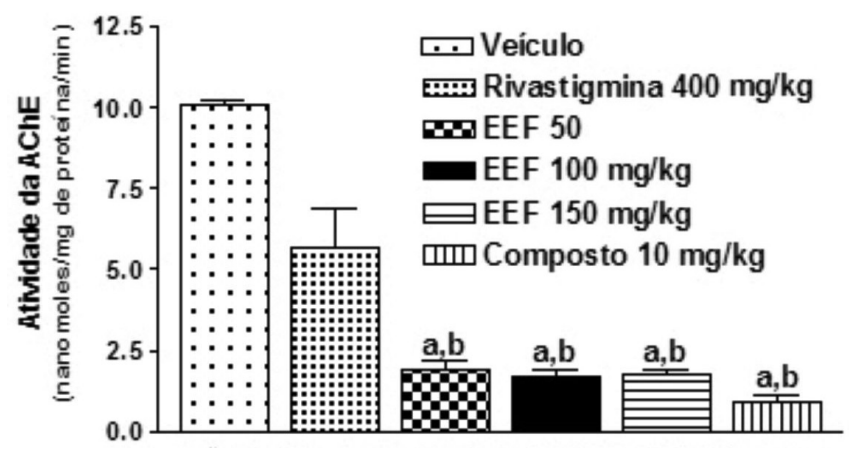

Figura 4. Efeito da fração enriquecida com flavonoides (composto) e EEF sobre a atividade da enzima acetilcolinesterase (AChE) no hipocampo de camundongos. Os grupos foram tratados conforme descritos no protocolo experimental $(n=7$ por grupo). Os valores representam a média \pm E.P.M. do número de animais usados nos experimentos. A atividade da acetilcolinesterase foi determinada em $10 \mathrm{~mL}$ de homogenato. Para análise estatística foram usados ANOVA e teste $t$-Student-Neuman-Keuls como post-hoc test. ${ }^{a}$ quando comparado ao veículo (solução salina $0,9 \% ; p<0,05$ ); ${ }^{b}$ quando comparado ao controle positivo (rivastigmina $400 \mathrm{mg} / \mathrm{kg}$; $p<0,05$ ). $E E F=$ extrato etanólico das flores

Os resultados sugerem que o EEF de $B$. perennis e o flavonoide isolado tem potencial para o uso no tratamento clínico de doenças neurodegenerativas, como a doença de Alzheimer, uma vez que a mesma diminuiu a atividade da $\mathrm{AChE}$ in vivo de forma significativa.

\section{CONCLUSÕES}

A fração flavonoídica isolada de EEF de $B$. perennis, contendo isorhamnetina 3-O- $\beta$-D-(6"-acetil)-galactopiranosideo, mostrou atividade anticolinesterásica in vitro. Sugere-se que o flavonoide pode aumentar de forma mais eficaz a estimulação colinérgica e, portanto, produzir um efeito inseticida mais potente do que outros inseticidas, como carbamatos e organofosforados, para aplicação na agropecuária ou em ambientes domésticos.

Nos estudos in vivo, verificou-se também uma diminuição da atividade enzimática da acetilcolinesterase quando se administrou nos animais EEF ou o flavonoide, sugerindo possível utilização no tratamento de doenças neurodegenerativas, como a Doença de Alzheimer.

A substância isolada poderá ser utilizada, após mais estudos, em formulação de produto(s) farmacêutico(s) para prevenção e/ou tratamento da Doença de Alzheimer, patologia associada à redução de neurotransmissores cerebrais, principalmente acetilcolina.

\section{MATERIAL SUPLEMENTAR}

Em http://quimicanova.sbq.org.br encontra-se a Figura 1S Espectros no ultravioleta registrados no DAD dos picos eluídos no cromatograma da fração isolada de EEF, em arquivo pdf e com acesso livre.

\section{AGRADECIMENTOS}

Ao Conselho Nacional de Desenvolvimento Científico e Tecnológico (CNPq) e à FAPEPI pelo apoio financeiro.

\section{REFERÊNCIAS}

1. Veiga Jr, V. F.; Maciel, M. A. M.; Pinto, A. C.; Quim. Nova 2005, 28, 519; Campelo, L. M. L.; Gonçalves, F. B. M.; Feitosa, C. M.; Freitas, R. M.; Pharm. Biol. 2011, 49, 709.

2. Judd, W. S.; Campbell, C. S.; Kellogg, E. A.; Stevens, P. F.; Donoghue, M. J.; Sistemática Vegetal: um enfoque filogenético, Artmed: Porto Alegre, 2009; Morikawa, T.; Li, X.; Nishida, E.; Nakamura, S.; Ninomiya, K.; Matsuda, H.; Hamao, M.; Muraoka, O.; Hayakawa, T.; Yoshikawa, M.; Chem. Pharm. Bull. 2011, 59, 889.

3. Avato, P.; Tava, A.; Phytochemistry 1995, 40, 141.

4. Oberbaum, M.; Galoyan, N.; Lerner-geva, L.; Singer, R.; Grisaru, S.; Shashar, D.; Samueloff, A.; Complement. Ther. Med. 2005, 13, 87; Morikawa, T.; Li, X.; Nishida, E.; Nakamura, S.; Ninomiya, K.; Matsuda, H.; Oda, Y.; Muraoka, O.; Yoshikawa, M.; J. Nat. Prod. 2008, 71, 828; Kavalcioğlu, N.; Açik, L.; Demirci, F.; Demirci, B.; Demir, H.; Baser, K. H.; Nat. Prod. Commun. 2010, 5, 147; Marques, T. H. C.; Cardoso, K. M. F.; Almeida, A. A. C.; Tomé, A. R.; Freitas, R. M.; Bol. Latinoam. Caribe Plant. Med. Aromat. 2011, 10, 338; Karakaş, F. P.; Karakaş, A.; Boran, C.; Türker, A. U.; Yalçin, F. N.; Bilensoy, E.; Pharm. Biol. 2012, 50, 1031.

5. Feitosa, C. M.; Luz, N. N.; Freitas, R. M.; Bezerra, M. Z. B.; Trevisan, M. T. S.; Braz. J. Biol. 2011, 71, 783.

6. Minett, T. S. C.; Bertolucci, P. H. F.; Rev. Neurociências 2000, 8, 11.

7. Viegas Jr, C.; Bolzani, V. S.; Furlan, M.; Fraga, C. A. M.; Barreiro, E. J.; Quim. Nova 2004, 27, 655.

8. Hueb, T. O.; Rev. Bras. Med. 2008, 65, 90; Smith, M. A. C.; Rev. Bras. Psiquiatria Genética 1999, 21, 4.

9. Sereniki, A.; Vital, M. A. B. F.; Rev. Psiquiatr. 2008, 30(1 Supl).

10. Mcnaughton, N.; Gray, J. A.; J. Affective Disord. 2000, 61, 161; Brandão, M. L.; Psicofisiologia: As bases fisiológicas do comportamento, $2^{\mathrm{a}}$ ed., Editora Atheneu: São Paulo, 2001, cap. 7, p. 125.

11. Recuero, M.; Vicente, M. C.; Martínez-García, A.; Ramos, M. C.; Carmona-saez, P.; Sastre, I.; Aldudo, J.; Vilella, E.; Frank, A.; Bullido, M. J.; Valdivieso, F.; Aging Cell 2009, 8, 128.

12. Honchar, M. P.; Olney, J. W.; Sherman, W. R.; Science 1983, 220, 323; Meldrum, B.; Garthwaite, J.; Trends Pharmacol. Sci. 1990, 11, 379; Marinho, M. M. F.; Sousa, F. C. F.; Bruin, V. M. S.; Vale, M. R.; Viana, G. S. B.; Neurochem. Int. 1998, 33, 299; Bannerman, D. M.; Rawlins, J. N.; McHugh, S. B.; Deacon, R. M.; Yee, B. K.; Bast, T.; Zhang, W. N.; Pothuizen, H. H.; Feldon, J.; J. Neurosci. Biobehav. Rev. 2004, 28, 273.

13. Ellman, G. E.; Coutney D.; Andres Junior, V.; Featherstone, R. M.; Biochem. Pharmacol. 1961, 7, 88.

14. Moyo, M.; Ndhlala, A. R.; Finnie, J. R.; Staden, J. V.; Food Chem. 2010, 123, 69.

15. Lowry, H.; Rosebrough, N. J.; Farr, A. L.; Randall, R. J.; J. Biol. Chem. 1951, 193, 265.

16. Yoshikawa, M.; Li, X.; Nishida, E.; Nakamura, S.; Matsuda, H.; Muraoka, O.; Morikawa, T.; Chem. Pharm. Bull. 2008, 56, 559.

17. Gudej, J.; Nazaruk, J.; Fitoterapia 2001, 72, 839.

18. Khan, M. T. H.; Orhan, I.; Senol, K. B. S.; Dvorska, M.; Chem. Biol. Interact. 2009, 181, 383.

19. Golan, D.; Tashjian, A. H.; Armstrong, E. J.; Armstrong, A.; Principles of Pharmacology: The Pathophysiologic Basis of Drug Therapy, $3^{\text {rd }}$ ed., Lippincott Williams \& Wilkins: Baltimore, 2011, chap. 2, 18. 


\section{ATIVIDADE ANTICOLINESTERÁSICA E PERFIL QUÍMICO DE UMA FRAÇÃO CROMATOGRÁFICA ATIVA DO EXTRATO ETANÓLICO DAS FLORES Bellis perennis L. (Asteraceae)}

Thiago Henrique Costa Marques, Pauline Sousa dos Santos e Rivelilson Mendes de Freitas*

Departamento de Bioquímica e Farmacologia, Centro de Ciências da Saúde, Universidade Federal do Piauí, 64049-550 Teresina - PI, Brasil

Rusbene Bruno Fonseca de Carvalho e Cassio Herbert Santos de Melo

Departamento de Química, Centro de Ciências da Natureza, Universidade Federal do Piauí, 64049-550 Teresina - PI, Brasil Juceni Pereira David

Faculdade de Farmácia, Universidade Federal da Bahia, 40170-115 Salvador - BA, Brasil Jorge Mauricio David e Luciano Silva Lima

Instituto de Química, Universidade Federal da Bahia, 40170-115 Salvador - BA, Brasil
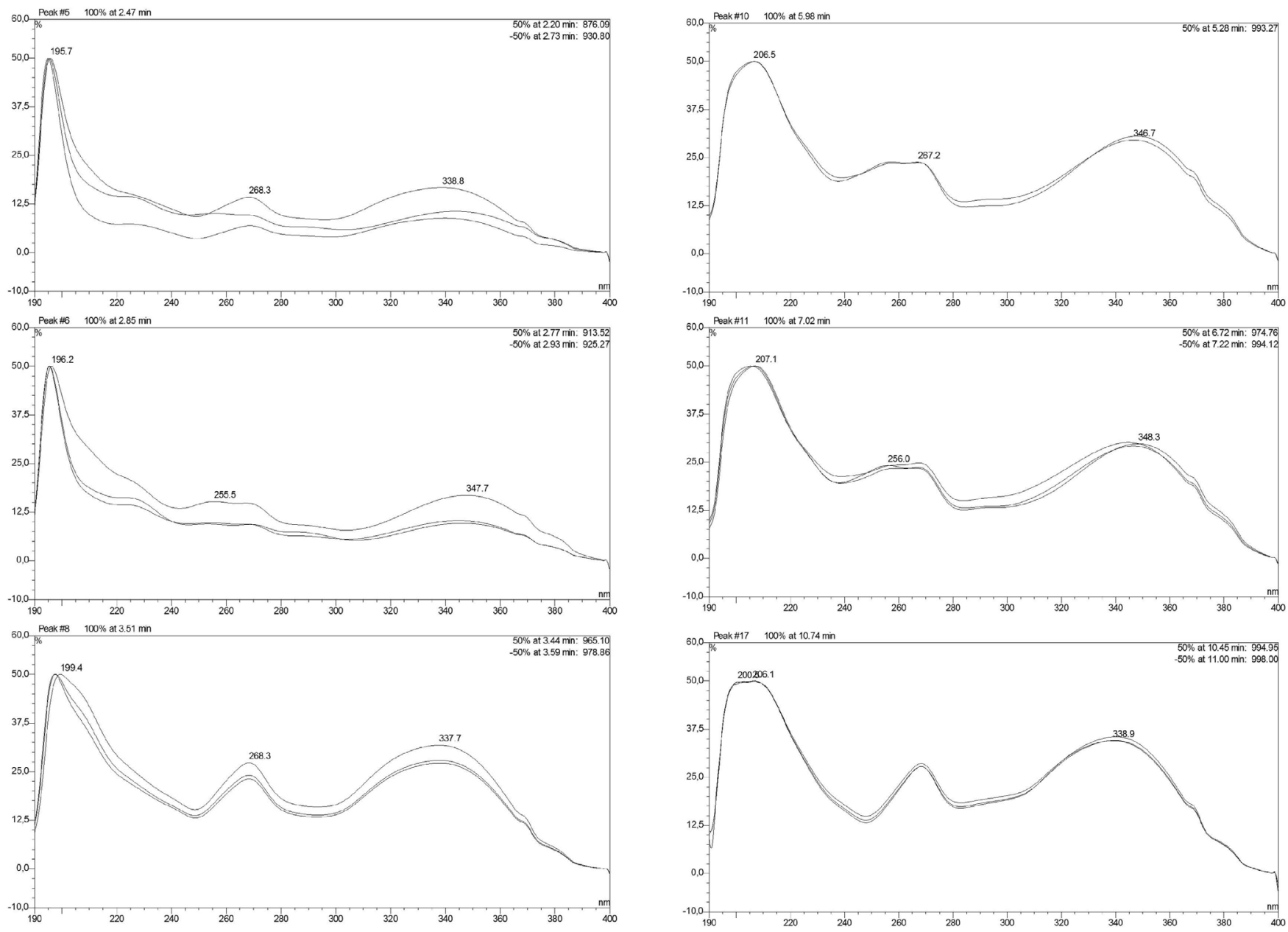

Figura 1S. Espectros no ultravioleta registrados no DAD dos picos eluídos no cromatograma da fração isolada de EEF 